\title{
REVIEW
}

\section{Cyr61 is a potential prognostic marker for prostate cancer}

\author{
Naoki Terada ${ }^{1}$, Prakash Kulkarni ${ }^{1,2}$ and Robert H Getzenberg ${ }^{1,2,3}$ \\ Cysteine-rich angiogenic inducer 61 (Cyr61) is an extracellular matrix protein involved in the transduction of growth factor and \\ hormone signaling that is frequently altered in expression in several types of cancers. In prostate cancer (PCa), Cyr61 is highly \\ expressed in organ-confined disease. Further, Cyr61 expression levels are associated with a lower risk of disease recurrence, and can be \\ quantitatively measured in the serum. Considered together, these results indicate that Cyr61 is a potential and clinically useful tissue, \\ as well as serum-based biomarker for differentiating lethal and non-lethal PCa.
}

Asian Journal of Andrology (2012) 14, 405-408; doi:10.1038/aja.2011.149; published online 20 February 2012

Keywords: Cyr61; lethal prostate cancer; non-lethal prostate cancer; overdiagnosis; overtreatment; prostate cancer

\section{CCN FAMILY PROTEINS}

Cysteine-rich angiogenic inducer 61 (Cyr61) is a member of the CCN family of proteins. Members of this family were first identified as secreted proteins whose synthesis was induced by mitogenic growth factors or by oncogene products. The first three members of the family were described as, Cyr61 (cystein-rich 61; CCN1), ${ }^{1}$ Ctgf (connective tissue growth factor; CCN2) ${ }^{2}$ and Nov (nephroblastoma overexpressed; CCN3), ${ }^{3}$ providing the acronym for the CCN family. CCN4 (Wisp1), CCN5 (Wisp2) and CCN6 (Wisp3) were subsequently identified as Wnt-inducible secreted proteins, ${ }^{4}$ and together they comprise the family of six homologous, cystein-rich proteins in vertebrates. $\mathrm{CCN}$ proteins share a modular structure, with an $\mathrm{N}$-terminal secretory peptide followed by four conserved domains with sequence homologies to insulin-like growth factor binding proteins, von Willebrand factor type $\mathrm{C}$ repeat, thrombospondin type I repeat and a carboxylterminal domain that contains a cysteine knot motif. ${ }^{5}$ More recently, members of the CCN family have emerged as dynamically expressed, extracellular matrix-associated proteins that play critical roles in cardiovascular and skeletal development, injury repair, fibrotic disease and cancer. In addition to mitogens and oncogenes, the synthesis of CCN proteins is highly inducible by serum growth factors, cytokines and environmental stress, such as hypoxia, UV exposure and mechanical stretching. The CCN proteins appear to function primarily via specific integrin receptors and heparin sulfate proteoglycans, thereby triggering signal transduction events that culminate in the regulation of cell adhesion, migration, proliferation, gene expression, differentiation and survival. ${ }^{6}$

\section{POSITIVE AND NEGATIVE ASSOCIATION BETWEEN CYR61 AND CANCER PROGRESSION}

Cyr61 signals through interaction with integrins, but downstream effects vary greatly depending on the combination of integrins bound. ${ }^{7}$ Previous data suggest that Cyr61 (CCN1) acts as a tumor-promoting factor and a key regulator in cancer progression, while Nov (CCN3) exhibits suppressive capabilities. ${ }^{7}$ However, more recently, numerous studies demonstrate altered Cyr61 expression in various cancers, depending on the cancer type Cyr61, may enhance or inhibit tumor growth. ${ }^{7-11}$

Consistent with its tumor-promoting function, Cyr61 was shown to be highly expressed in breast cancer. ${ }^{12}$ Moreover, there was significant association between Cyr61 expression and stage, tumor size, positive lymph nodes, age and estrogen receptor status, linking the expression of Cyr61 to clinical and pathological parameters. ${ }^{13}$ This underlines a role for Cyr61 in the progression of breast cancer and suggests that it could serve as a valuable tool for monitoring tumor status. Furthermore, Cyr61 levels are increased in MCF-7 breast cancer cells upon stimulation by estrogens, suggesting that the corresponding protein may be involved in the development of estrogen-mediated breast cancer. ${ }^{13}$ It was also demonstrated that Cyr61 is sufficient for estrogen independence and anti-estrogen resistance, to promote invasiveness in vitro and to induce tumorigenesis and neovascularization in vivo. ${ }^{14}$

Cyr61 was also shown to be highly expressed in primary gliomas, both in cell lines derived from high-grade gliomas and in the more tumorigenic astrocytomas. ${ }^{15}$ As was seen with breast tumors, a correlation was found between Cyr61 expression and tumor grade, pathology, gender, age at diagnosis and survival of glioma patients. The detection of Cyr61 at the time of diagnosis is of prognostic significance and suggests that it may play a role in tumor progression. ${ }^{16}$ Indeed, stable expression of Cyr61 in gliomas enhances anchorage independent cell growth and cell migration, and formed more tumors than control cells when injected in nude mice. ${ }^{16}$

On the other hand, Cyr61 has been described as antiproliferative and antitumorigenic agent in non-small cell lung cancer where its expression was markedly decreased in human lung tumor samples. ${ }^{17}$ Furthermore, transfection of Cyr61 in Cyr61-negative cell lines 
resulted in a reduced number of cells able to grow in an anchorageindependent fashion. ${ }^{17}$

\section{CYR61 EXPRESSION IN PROSTATE CANCER (PCA)}

Kulkarni et al. ${ }^{18}$ examined the gene expression profiles by DNA microarray analysis of 10 normal prostate, five asymptomatic benign prostatic hyperplasia (BPH), eight symptomatic $\mathrm{BPH}$ and eight $\mathrm{BPH}$ with $\mathrm{PCa}$ samples. In this study, the expression levels in Cyr61 were higher in $\mathrm{BPH}$ with $\mathrm{PCa}$ than those without PCa. All these $\mathrm{BPH}$ with PCa samples were obtained by radical prostatectomy from patients with Gleason score 6/7 and stage T2/T3 PCa without metastasis. These results were validated by real-time PCR using other sample sets. Cyr61 expression ratios compared to TATA-binding protein were significantly higher in PCa tissues $(n=10,9.05 \pm 5.44)$ than in donor prostate tissues $(n=10$, $1.84 \pm 1.34)(P<0.05) .{ }^{19}$ In contrast to these results, a previous study showed that Cyr61 mRNA expression was downregulated in PCa tissue when compared to normal tissue adjacent to the cancer lesion. ${ }^{20}$ In this study, most of patients had advanced PCa with pathological stage of T3 or T4 with lymph node metastasis. Thus, we queried the ONCOMINE database to interrogate Cyr61 expression in multiple sample sets. ${ }^{21}$ In two studies, Cyr61 was upregulated in PCa tissue when compared to healthy prostate tissue and prostatic intraepithelial neoplasia (PIN) tissue, and in nine studies in which expression was compared in primary and metastatic PCa samples, Cyr61 expression was lower in metastases than in the primary lesion. From these observations resulting from a meta-analysis, it can be concluded that Cyr61 mRNA expression is higher in localized PCa than in advanced, metastatic disease.

Taken together, the Cyr61 expression patterns are atypical in that they are low in normal tissue, increased in localized disease and then again low in metastatic cancer. As a micro-environmental regulator, Cyr61 may have differential effects on local disease compared with distant metastasis. Function of Cyr61 appears to be complex and dependent on cell types. In PCa cells, Cyr61 expression is low in LNCaP, moderate in DU145 and high in PC3, correlating with their aggressiveness. Moreover, it was reported that knocking down Cyr61 expression suppresses cell migration in DU145 and PC3, invasion and proliferation and that Cyr61 regulates Rac1 signaling, a mechanism by which Cyr61 may exert its influence on growth and motility. ${ }^{22}$ It was also reported that knockdown of Cyr61 expression in LNCaP, DU145 and PC3 cells severely blunted their sensitivity to TRAIL, an effect that was reversed by exogenously added Cyr61 protein, showing that Cyr61 contributes to TRAIL-induced apoptosis in PCa cells. ${ }^{23}$ These results indicate that Cyr61 appears to be a double-edged sword for PCa cells and could partially explain the mechanisms underlying the biphasic changes in Cyr61 expression during PCa progression. However, further analyses are needed to illustrate the exact function of Cyr61 in PCa.

\section{CYR61 AS A DIAGNOSTIC TISSUE MARKER}

The accuracy of the pathologic diagnosis of PCa is critical for optimal patient care. Even though the diagnosis can usually be made on morphologic features such as growth pattern, nuclear atypia and the absence of basal cells, it is sometimes difficult to reach a firm diagnosis by routine histological study of hematoxylin and eosin-stained biopsy tissue, in particular for small foci of cancer. ${ }^{24}$ No single morphologic feature is cancer specific and many benign conditions can mimic PCa. Therefore, the application of immunohistochemistry (IHC) to distinguish PCa from benign lesions and to confirm the diagnosis becomes helpful and necessary, especially in equivocal cases.

The enzyme $\alpha$-methylacyl-coenzyme A racemase (AMACR) is a biomarker that was identified by both differential display and expression array analysis as a gene abundantly expressed in PCa relative to benign prostate epithelium. ${ }^{25,26}$ In a meta-analysis of four DNA microarray data sets, AMACAR was one of the genes most consistently overexpressed in $\mathrm{PCa} .{ }^{27}$ In IHC analysis AMACR was characteristically upregulated in $80 \%-100 \%$ of PCa tissues. ${ }^{28}$ Hence, AMACR is considered to be a useful IHC marker for PCa. However, this marker also stains up to $21 \%$ of benign prostatic glands and $79 \%$ of partial atrophy lesions. ${ }^{29}$ Therefore, AMACR positivity must be evaluated with caution.

To adjudicate Cyr61 as a diagnostic tissue biomarker for PCa, we performed IHC on tissue microarrays (TMA) containing 1366 spots representing 197 prostates from consecutive radical prostatectomy specimens (Figure 1). ${ }^{19}$ In comparison to the normal prostate, Cyr61 staining intensity was significantly higher in atrophy $(n=40$, $P=0.0009)$, PIN $(n=25, P<0.0001)$ and PCa $(n=174, P<0.0001)$. However, its expression levels were not significantly different between the 23 PIN and matched PCa lesions $(P=0.06) .{ }^{19}$ These results indicated that Cyr61 is likely important for PCa development even in atrophy or PIN. Our study shows similarities between Cyr61 and AMACR, particularly increased expression of the respective proteins in PCa versus histologically normal adjacent tissue or normal donor prostate tissue. Further examinations are needed for analyzing the association between Cyr61 and AMACR. However, IHC evaluation for Cyr61 expression has a potential to aid in diagnosing problematic atypical cases.

\section{CYR61 AS A PROGNOSTIC TISSUE MARKER}

In addition to its potential utility in discerning PCa from benign prostatic tissue, it was reported that AMACAR expression is lower in metastatic PCa than in localized $\mathrm{PCa}^{30,31}$ and that AMACR expression levels in localized PCa are negatively associated with biochemical recurrence and cancer-specific death. ${ }^{32}$ Thus, we evaluated the association between Cyr61 staining intensity and subsequent recurrence after surgical treatment of localized PCa. A study of 229 men with recurrence and 229 controls matched on age, race, pathologic stage and Gleason sum, utilizing IHC analysis of TMA sections, was conducted. Cyr61 staining intensity was not associated with Gleason sum or presurgery prostate-specific antigen (PSA) levels, but the proportion of T3b or worse disease was more common in TMA spots with higher staining intensity than in those with low staining intensity. Cyr61 staining intensity in TMA spots containing cancer was not linearly associated with risk of recurrence, which was lowest in men with at least 1 TMA spot containing cancer with a staining intensity of 3 when compared with 0 . However, $12.2 \%$ of cases and $24.0 \%$ of controls had at least 1 TMA spot containing cancer with a staining intensity of 3 , a difference that was statistically significant $(P=0.001)$. Taking into consideration age, pathologic stage and grade, presurgery PSA concentrations, and calendar of surgery as a measure of tissue block storage time, men with Cyr61 staining intensity of 3 were $56 \%$ less likely to recur than men with a lower staining intensity (odds ratio: $0.44 ; 95 \%$ confidence interval: $0.22-0.90$ ). Therefore, it was concluded that decreased expression of Cyr61 was associated with PCa recurrence after surgical treatment. ${ }^{33}$

It is interesting that Cyr61 correlates with improved prognosis in $\mathrm{PCa}$, but a worse prognosis in breast cancer. ${ }^{34}$ On the surface, these appear to be divergent findings in these two hormonally sensitive tumor types. The tumor micro-environment has been associated with cancer progression. ${ }^{35}$ Cyr61 is regulated by various growth factors, such as epidermal growth factor, basic fibroblast growth factor, or transforming growth factor- $\beta$ and chemokines, such as IL-1, IL-2 or 




Figure 1 Immunohistochemistry reveals Cyr61 protein expression in prostate tissues: (a) normal prostate tissue, (b) BPH, (c) PIN, (d) tumor, (e) tumor and normal prostate. This figure is reproduced from D'Antonio et al. ${ }^{19} \mathrm{BPH}$, benign prostatic hyperplasia; Cyr61, cysteine-rich angiogenic inducer 61; PIN, prostatic intraepithelial neoplasia.

IL-6. It is also inducible by serum cortisol, estrogen, tumor necrosis factor- $\alpha$ and prostaglandins. ${ }^{36}$ These signals either suppress or promote tumors depending on the cell type. We found that Cyr61 expression in PCa was modulated by oncogenic 'stimulation' such as androgens or lysophosphatidic acid, as well as anti-oncogenic 'stress' such as serum starvation or phosphoinositide 3-kinase inhibition in the tumor environment. ${ }^{37}$ These results suggest that differences in Cyr61 expression may reflect the extracellular environmental status of the cancer, which is distinct between PCa and breast cancer.

\section{CYR61 AS A SERUM MARKER}

Cyr61 is an extracellular matrix protein that is secreted and therefore, represents an attractive serum biomarker candidate. To explore this possibility, we first established a sandwich ELISA to measure Cyr61 concentrations in cell supernatants. ${ }^{38}$ The concentration of Cyr61 in the medium alone was $1.1 \mathrm{ng} \mathrm{ml}^{-1}$ and those in the supernatants containing 80\% confluent LNCaP, DU145 and PC3 cells were 4.0, 33.5 and $215.1 \mathrm{ng}$ $\mathrm{ml}^{-1}$, respectively, indicating that the Cyr61 protein secreted by the PCa cells could be measured by the sandwich ELISA. ${ }^{37}$ Cyr61 levels were then measured in serum samples obtained from organ-confined (OC)-PCa $(n=58)$ and non-organ-confined (NOC)-PCa $(n=57)$ patients. Cyr61 levels were significantly higher in NOC-PCa $\left(116.3 \pm 140.2 \mathrm{ng} \mathrm{ml}^{-1}\right)$ than in OC-PCa $\left(79.7 \pm 56.0 \mathrm{ng} \mathrm{ml}^{-1}\right)$, although PSA levels were not significantly different $\left(6.4 \pm 3.8 \mathrm{ng} \mathrm{ml}^{-1}\right.$ in OC-PCa and $6.1 \pm 4.7 \mathrm{ng} \mathrm{ml}^{-1}$ in NOC-PCa, $P=0.376$ ) (Figure 2a). ${ }^{37}$ Serum Cyr61 levels are not correlated with serum PSA levels and may increase the accuracy for differentiating NOC-PCa from OC-PCa combined with serum PSA levels and other clinical parameters used in PCa staging nomograms. ${ }^{39}$ However, serum Cyr61 levels tended to be higher in control patients compared with OC-PCa patients. This may be due to the fact that in these control patients, PSA levels are high although their prostate biopsies were found to be negative, suggesting that inflammation in their prostate tissues
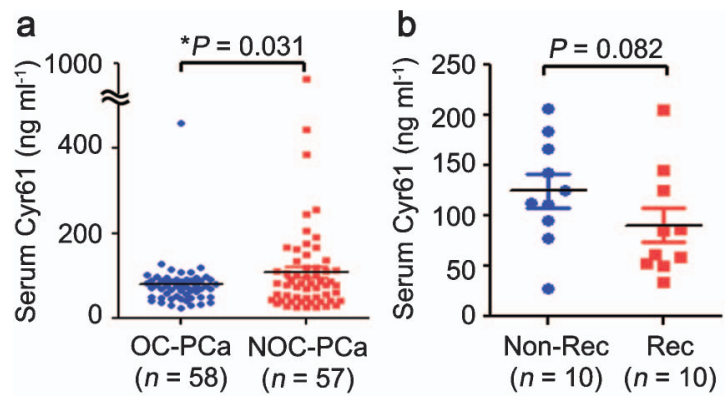

Figure 2 Serum Cyr61 levels measured by sandwich ELISA. (a) Cyr61 concentrations in serum samples obtained from OC-PCa $(n=58)$ and NOC-PCa $(n=57)$ patients, demonstrating significant difference ( $* P=0.031$ ). (b) Cyr61 concentrations in serum samples obtained from $\mathrm{PCa}$ patients without PSA recurrence (Non-Rec, $n=10$ ) and those with PSA recurrence (Rec, $n=10$ ), demonstrating no significant difference $(P=0.082)$. This figure is reproduced from D'Antonio et al. ${ }^{19}$ Cyr61, cysteine-rich angiogenic inducer 61 ; NOC, non-organ-confined; $\mathrm{OC}$, organ-confined; PCa, prostate cancer.

might have induced PSA production. ${ }^{40}$ Interestingly, Cyr61 expression in benign prostatic cells was enhanced by treating with prostaglandin E2 or transforming growth factor- $\beta .^{41}$ Therefore, it is quite possible that inflammation in benign prostate tissues induced Cyr61 production in control patients. Pretreatment knowledge of OC-PCa or NOC$\mathrm{PCa}$ is important for treatment selection and planning. However, it was observed that approximately $10 \%$ of OC-PCa patients and $50 \%$ of NOC-PCa patients experienced disease progression within 10 years after surgery. ${ }^{42,43}$ It is challenging to find serum markers that can identify patients with disease progression preoperatively. To further address the question of whether or not serum Cyr61 levels could predict disease progression after surgery, we collected serum samples from PCa patients that later recurred (Rec, $n=10)$ and those that did not recur within a similar time frame (non-Rec, $n=10)$. The serum Cyr61 levels in the Rec patients $\left(90.5 \pm 17.0 \mathrm{ng} \mathrm{ml}^{-1}\right)$ tended to be lower than in the non-Rec patients $\left(125.0 \pm 16.7 \mathrm{ng} \mathrm{ml}^{-1}\right)$, although these differences were not statistically significant $(P=0.082)$ (Figure $2 \mathbf{b}) .^{37}$ These results indicate that $\mathrm{PCa}$ patients with lower serum Cyr61 levels might have a higher risk of PSA recurrence. However, additional studies with larger patient cohorts will be required to elucidate whether serum Cyr61 levels can predict PCa recurrence.

\section{CONCLUSIONS}

Cyr61 expression increases during PCa development and decreases as the disease progresses (Figure 3). Cyr61 is highly expressed even in

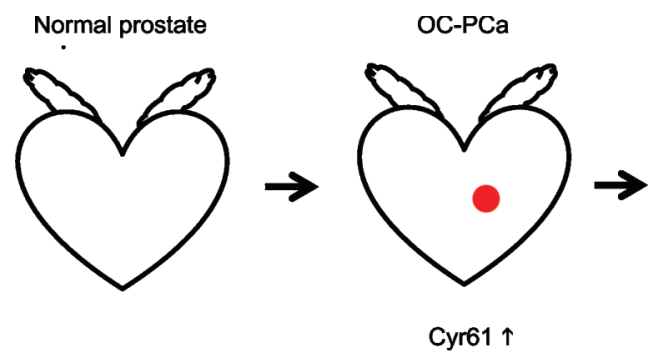

Low grade PCa with high Cyr61
NOC-PCa

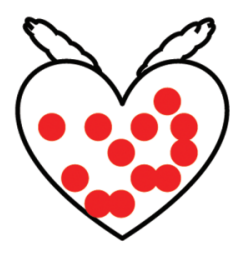

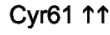

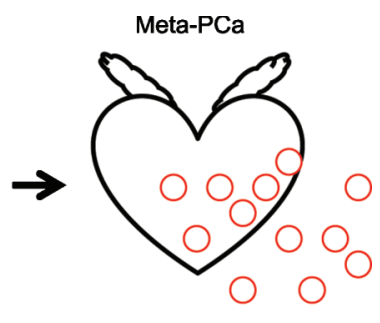

Cyr61 $\downarrow$

Low grade PCa with low Cyr61

Figure 3 Biphasic changes in Cyr61 expression during prostate cancer progression. Filled circles show low-grade PCa cells with high Cyr61 expression and unfilled circles show high-grade PCa cells with low Cyr61 expression. Cyr61 expression increases during PCa development and local progression. However, it decreases during the progression into meta-PCa. These tumor cells secrete Cyr61 protein into serum and circulating Cyr61 levels are a potential serum-based biomarker for characterizing PCa. Cyr61, cysteine-rich angiogenic inducer 61; meta-PCa, metastatic prostate cancer; NOC, non-organ-confined; OC, organ-confined. 
PIN or early stage PCa and is a useful tissue biomarker for the detection of PCa in biopsy samples. On the other hand, Cyr61 expression levels in radical prostatectomy samples are negatively correlated with PCa recurrence and are a useful marker for predicting patient prognosis. The serum Cyr61 levels are higher in NOC-PCa patients than in OC-PCa patients, but tend to be lower in patients with disease recurrence. These results are consistent to the Cyr61 expression levels in PCa tissues. Serum Cyr61 levels are not correlated with serum PSA levels and may have a potential to increase the accuracy for differentiate lethal and non-lethal PCa.

\section{COMPETING FINANCIAL INTERESTS}

The authors declare no competing financial interests.

\section{ACKNOWLEDGMENTS}

This paper is supported by the NCI Prostate Cancer SPORE grant (P50 CA058236), the Patana Fund of the Brady Urological Institute, and a grant from the Patrick C Walsh Fund of the Brady Urological Institute. We thank Dr Alan Partin, Ms. Leslie Mangold and Dr Mario Eisenberger (The James Buchanan Brady Urological Institute, The Johns Hopkins University School of Medicine) for sample collection and members of the Getzenberg lab for many helpful discussions.

1 O'Brien TP, Yang GP, Sanders L, Lau LF. Expression of Cyr61, a growth factorinducible immediate-early gene. Mol Cell Biol 1990; 10: 3569-77.

2 Bradham DM, Igarashi A, Potter RL, Grotendorst GR. Connective tissue growth factor: a cysteine-rich mitogen secreted by human vascular endothelial cells is related to the SRC-induced immediate early gene product CEF-10. J Cell Biol 1991; 114: 128594.

3 Joliot V, Martinerie C, Dambrine G, Plassiart G, Brisac M et al. Proviral rearrangements and overexpression of a new cellular gene (Nov) in myeloblastosis-associated virus type 1-induced nephroblastomas. Mol Cell Biol 1992; 12: 10-21.

4 Pennica D, Swanson TA, Welsh JW, Roy MA, Lawrence DA et al. WISP genes are members of the connective tissue growth factor family that are up-regulated in Wnt1 -transformed cells and aberrantly expressed in human colon tumors. Proc Natl Acad Sci USA 1998; 95: 14717-22.

5 Bork P. The modular architecture of a new family of growth regulators related to connective tissue growth factor. FEBS Lett 1993; 327: 125-30.

6 Chen CC, Lau LF. Functions and mechanisms of action of CCN matricellular proteins. Int J Biochem Cell Biol 2009; 41: 771-83.

7 Bleau AM, Planque N, Perbal B. CCN proteins and cancer: two to tango. Front Biosci 2005; 10: 998-1009.

8 Lin MT, Zuon CY, Chang CC, Chen ST, Chen CP et al. Cyr61 induces gastric cancer cell motility/invasion via activation of the integrin/nuclear factor-kappaB/cyclooxygenase2 signaling pathway. Clin Cancer Res 2005; 11: 5809-20.

9 Feng P, Wang B, Ren EC. Cyr61/CCN1 is a tumor suppressor in human hepatocellular carcinoma and involved in DNA damage response. Int J Biochem Cell Biol 2008; 40: 98-109.

10 Holloway SE, Beck AW, Girard L, Jaber MR, Barnett CC Jr et al. Increased expression of Cyr61 (CCN1) identified in peritoneal metastases from human pancreatic cancer. J Am Coll Surg 2005; 200: 371-7.

11 Chien W, Kumagai T, Miller CW, Desmond JC, Frank JM et al. Cyr61 suppresses growth of human endometrial cancer cells. J Biol Chem 2004; 279: 53087-96.

12 Tsai MS, Hornby AE, Lakins J, Lupu R. Expression and function of CYR61, an angiogenic factor, in breast cancer cell lines and tumor biopsies. Cancer Res 2000; 60: 5603-7.

13 Xie D, Miller CW, O'Kelly J, Nakachi K, Sakashita A et al. Breast cancer. Cyr61 is overexpressed, estrogen-inducible, and associated with more advanced disease. J Biol Chem 2001; 276: 14187-94.

14 Menendez JA, Mehmi I, Griggs DW, Lupu R. The angiogenic factor CYR61 in breast cancer: molecular pathology and therapeutic perspectives. Endocr Relat Cancer 2003; 10: 141-52.

15 Xie D, Yin D, Tong X, O'Kelly J, Mori A et al. Cyr61 is overexpressed in gliomas and involved in integrin-linked kinase-mediated Akt and beta-catenin-TCF/Lef signaling pathways. Cancer Res 2004; 64: 1987-96.

16 Xie D, Yin D, Wang HJ, Liu GT, Elashoff R et al. Levels of expression of CYR61 and CTGF are prognostic for tumor progression and survival of individuals with gliomas. Clin Cancer Res 2004; 10: 2072-81.
17 Tong X, Xie D, O'Kelly J, Miller CW, Muller-Tidow C et al. Cyr61, a member of CCN family, is a tumor suppressor in non-small cell lung cancer. J Biol Chem 2001; 276: 47709-14.

18 Prakash K, Pirozzi G, Elashoff M, Munger W, Waga I et al. Symptomatic and asymptomatic benign prostatic hyperplasia: molecular differentiation by using microarrays. Proc Natl Acad Sci USA 2002; 99: 7598-603.

19 D'Antonio KB, Toubaji A, Albadine R, Mondul AM, Platz EA et al. Extracellular matrix associated protein CYR61 is linked to prostate cancer development. J Urol 2010; 183: $1604-10$

20 Pilarsky CP, Schmidt U, Eissrich C, Stade J, Froschermaier SE et al. Expression of the extracellular matrix signaling molecule Cyr61 is downregulated in prostate cancer. Prostate 1998; 36: 85-91.

21 Rhodes DR, Yu J, Shanker K, Deshpande N, Varambally R et al. ONCOMINE: a cancer microarray database and integrated data-mining platform. Neoplasia 2004; 6: 1-6.

22 Sun ZJ, Wang Y, Cai Z, Chen PP, Tong XJ et al. Involvement of Cyr61 in growth, migration, and metastasis of prostate cancer cells. Br J Cancer 2008; 99: 1656-67.

23 Franzen CA, Chen CC, Todorovic V, Juric V, Monzon RI et al. Matrix protein CCN1 is critical for prostate carcinoma cell proliferation and TRAIL-induced apoptosis. $\mathrm{Mol}$ Cancer Res 2009; 7: 1045-55.

24 Varma M, Jasani B. Diagnostic utility of immunohistochemistry in morphologically difficult prostate cancer: review of current literature. Histopathology 2005; 47: 1-16.

25 Rubin MA, Zhou M, Dhanasekaran SM, Varambally S, Barrette TR et al. Alphamethylacyl coenzyme A racemase as a tissue biomarker for prostate cancer. JAMA 2002; 287: 1662-70.

26 Luo J, Zha S, Gage WR, Dunn TA, Hicks JL et al. Alpha-methylacyl-CoA racemase: a new molecular marker for prostate cancer. Cancer Res 2002; 62: 2220-6.

27 Rhodes DR, Barrette TR, Rubin MA, Ghosh D, Chinnaiyan AM. Meta-analysis of microarrays: interstudy validation of gene expression profiles reveals pathway dysregulation in prostate cancer. Cancer Res 2002; 62: 4427-33.

28 Jiang Z, Woda BA, Wu CL, Yang XJ. Discovery and clinical application of a novel prostate cancer marker: alpha-methylacyl CoA racemase (P504S). Am J Clin Pathol 2004; 122: 275-89.

29 Humphrey PA. Diagnosis of adenocarcinoma in prostate needle biopsy tissue. J Clin Pathol 2007; 60: 35-42

30 Kuefer R, Varambally S, Zhou M, Lucas PC, Loeffler M et al. Alpha-methylacyl-CoA racemase: expression levels of this novel cancer biomarker depend on tumor differentiation. Am J Pathol 2002; 161: 841-8.

31 Rubin MA, Zerkowski MP, Camp RL, Kuefer R, Hofer MD et al. Quantitative determination of expression of the prostate cancer protein alpha-methylacyl-CoA racemase using automated quantitative analysis (AQUA): a novel paradigm for automated and continuous biomarker measurements. Am J Pathol 2004; 164: 831-40.

32 Rubin MA, Bismar TA, Andren O, Mucci L, Kim R et al. Decreased alpha-methylacyl CoA racemase expression in localized prostate cancer is associated with an increased rate of biochemical recurrence and cancer-specific death. Cancer Epidemiol Biomarkers Prev 2005; 14: 1424-32.

33 D'Antonio KB, Schultz L, Albadine R, Mondul AM, Platz EA et al. Decreased expression of cyr61 is associated with prostate cancer recurrence after surgical treatment. Clin Cancer Res 2010; 16: 5908-13.

34 Xie D, Nakachi K, Wang H, Elashoff R, Koeffler HP. Elevated levels of connective tissue growth factor, WISP-1, and CYR61 in primary breast cancers associated with more advanced features. Cancer Res 2001; 61: 8917-23.

35 Karan D, Thrasher JB, Lubaroff D. Prostate cancer: genes, environment, immunity and the use of immunotherapy. Prostate Cancer Prostatic Dis 2008; 11: 230-6.

36 Chen Y, DuXY. Functional properties and intracellular signaling of CCN1/Cyr61. J Cell Biochem 2007; 100: 1337-45.

37 Terada N, Shiraishi T, Zeng Y, Mooney SM, Yeater DB et al. Cyr61 is regulated by cAMP-dependent protein kinase with serum levels correlating with prostate cancer aggressiveness. Prostate; e-pub ahead of print 24 October 2011; doi: 10.1002/ pros. 21501.

38 Sakamoto S, Yokoyama M, Prakash K, Tsuruha J, Masamoto S et al. Development of quantitative detection assays for CYR61 as a new marker for benign prostatic hyperplasia. J Biomol Screen 2003; 8: 701-11.

39 Partin AW, Mangold LA, Lamm DM, Walsh PC, Epstein JI et al. Contemporary update of prostate cancer staging nomograms (Partin Tables) for the new millennium. Urology 2001; 58: 843-8.

40 Kandirali E, Boran C, Serin E, Semercioz A, Metin A. Association of extent and aggressiveness of inflammation with serum PSA levels and PSA density in asymptomatic patients. Urology 2007; 70: 743-7.

41 Sakamoto S, Yokoyama M, Aoki M, Suzuki K, Kakehi Y et al. Induction and function of CYR61 (CCN1) in prostatic stromal and epithelial cells: CYR61 is required for prostatic cell proliferation. Prostate 2004; 61: 305-17.

42 Epstein JI, Pizov G, Walsh PC. Correlation of pathologic findings with progression after radical retropubic prostatectomy. Cancer 1993; 71: 3582-93.

43 Hull GW, Rabbani F, Abbas F, Wheeler TM, Kattan MW et al. Cancer control with radical prostatectomy alone in 1,000 consecutive patients. J Urol 2002; 167: 528-34. 